\title{
Análisis de la dimensión medioambiental de las compañías aereas que operan en España: estudio del contenido de sus páginas web
}

\author{
Diana Catalina PÉREZ-Bustamante YÁBAR \\ Beatriz RODRÍGUEZ HERRÁEZ \\ María Luisa MEDRANO GARCíA \\ Universidad Rey Juan Carlos
}

Recibido: 15 de Octubre de 2011

Enviado a evaluar: 16 de Octubre de 2011

Aceptado: 25 de Octubre de 2011

\begin{abstract}
RESUMEN
El turismo es un pilar fundamental en el desarrollo económico y social de España. Dentro del sector turístico, las compañías aéreas suponen un elemento de vital importancia, al ser este medio el más utilizado por los turistas para sus desplazamientos. Dichas compañías producen un elevado porcentaje de contaminación -atmosférica y acústica, fundamentalmente- que ha de reducirse porque su volumen y ritmo de evolución son insostenibles tanto desde una óptica global como desde la perspectiva de los objetivos de desarrollo sostenible y las políticas de protección ambiental de los países avanzados, especialmente, en los Estados miembros de la Unión Europea. Así pues, se observa en la actualidad cómo las compañías aéreas avanzan hacia el desarrollo de políticas de Responsabilidad Social Corporativa que enfatizan la dimensión medioambiental de su negocio. Analizar la dinámica empresarial de las mismas, desde esta perspectiva, es importante para extraer conclusiones acerca de la importancia del cambio estructural y funcional del sistema social, económico y político para la sostenibilidad y, también, para el mantenimiento de los equilibrios básicos del ecosistema socioeconómico y ambiental de nuestros días.
\end{abstract}

Palabras clave: Responsabilidad Social Corporativa; Acciones medioambientales, Análisis de contenido; Compañías aéreas; Páginas Webs; Política ambiental.

\section{Analysis of airlines operative in Spain environmental dimensions: a review of their website content}

\begin{abstract}
Tourism is a significant economic sector since the economic and social development of Spain points of view. Within, airlines are crucial due to it accounts for the highest share on international tourists transport mean selection. Airlines produce a significant percentage of total pollution -mostly atmospheric and acoustic, that should be reduced because their volume and rhythm of increase are unsustainable from the global
\end{abstract}


perspective and also for sustainable development aims and environmental protection policies, particularly the E.U policies. Therefore, airlines are progressing nowadays through Social Corporative Responsibility policies which emphasize their business environmental dimension. It is relevant to analyze those firm SCR dynamics in order to obtain conclusions on the structural and functional systems drivers of change - since the social economic and political perspectives and its relations to sustainability; also in terms to their collaboration to socioeconomic and environmental basic equilibrium maintenance.

Keywords: Social Corporative Responsibility; Environmental actions; Research analysis; Airline companies; Websites; Environmental Policy.

\section{INTRODUCCIÓN}

El turismo ha sido considerado en los últimos tiempos, un pilar fundamental en el desarrollo de la economía y la sociedad española. Este sector es crucial para España en la actualidad, puesto su ejecutoria es considerablemente mejor que la media de los sectores de actividad económica. En efecto, la evolución de las principales variables macroeconómicas revela con claridad que nuestro país está viviendo una etapa de estancamiento económico y, en consecuencia, alto nivel de desempleo y reducción considerable del bienestar, consistentes con el estancamiento de la reducción de la actividad productiva.

Las últimas cifras del Instituto Nacional de Estadística (INE) confirman la existencia de un panorama general complejo en España, con tasas de crecimiento del PIB muy reducidas $\left(0,7 \%\right.$ en el $2^{\circ}$ trimestre de 2011$)$ y una tasa de paro del $20,89 \%$ en septiembre de 2011. Por el contrario, el sector turístico (INE, 2011), muestra una realidad diferente.

Entre los meses de Enero y Agosto del año 2011 y, a pesar de la crisis económica y financiera mundial, España ha recibido un total de 39,9 millones de turistas internacionales, lo que supone 2,9 millones de turistas más que en el mismo periodo del año anterior y un crecimiento interanual del 7,8\% (IET, 2011a). Esta tasa de variación positiva supera la de crecimiento medio del turismo internacional en Europa, situada en el 6\% en el mismo periodo. Durante los siete primeros meses del año, los ingresos de España procedentes del turismo han ascendido a casi 29,5 mil millones de euros (IET, 2011b). Los últimos datos sobre la participación del turismo en el PIB han evolucionado en este mismo sentido y muestran una variación positiva, que se sitúa actualmente en el 10,23 por ciento (Figuerola, 2011). No obstante, hay que señalar que las tendencias a medio plazo de la participación del VAB del sector turístico en el PIB español indican una ligera pérdida de peso específico del mismo. De hecho, en el año 2001 la participación del PIB turístico en la economía española se situó en el 11,5\% y el ratio ha registrado un ligero descenso paulatino desde entonces, hasta situarse en el 10,14\%, en el año 2009.

Así pues, durante la fase alcista del ciclo económico y, en concreto durante la última década anterior a la quiebra de Lehman Brothers, la actividad turística en España había experimentado un menor dinamismo que el conjunto de la economía española (Exceltur, 2009). Ahora, en cambio, parece que estas tendencias se han invertido y la participación relativa del sector en el PIB vuelve a elevarse, cambio que sería conve- 
niente consolidar en los próximos meses. En todo caso, estos datos revelan que la importancia del sector turístico en la economía española sigue siendo incuestionable.

Dentro del sector turístico, las compañías aéreas juegan un papel de vital importancia al ser el avión el medio de transporte más utilizado por los turistas internacionales en sus viajes a España, accediendo por esta vía un 77 por ciento de los turistas (IET, 2010). Este hecho hace que el sector aeronáutico tenga una gran importancia en la economía española por su elevada contribución a la actividad del sector del turismo.

Por otro lado, la Responsabilidad Social Empresarial (RSE), también denominada Responsabilidad Social Corporativa (RSC) es un concepto muy utilizado actualmente, especialmente por las empresas de muchos sectores de actividad que presentan memorias, generan programas de formación para sus empleados bajo esta denominación, etc., y al que han dado importancia, también, especialistas de muy diversos ámbitos desde la educación a la política.

La Responsabilidad Social Empresarial se consideró en sus inicios como algo pasajero que no tendría cabida en los objetivos de las organizaciones, una actividad a la que los directivos no le otorgaban ninguna importancia. Pero el tiempo ha demostrado que estas afirmaciones eran equivocadas. En la actualidad es la sociedad la que demanda a las entidades un compromiso con todos los agentes relacionados directa o indirectamente con las empresas.

Como era de esperar finalmente el concepto y la exigencia de responsabilidad social empresarial ha llegado al ámbito de las compañías aéreas y se ha convertido es uno de los más complejos retos que ha de enfrentar la gestión empresarial de transporte de pasajeros a sus destinos turísticos. Al análisis de esta temática se dedica el presente trabajo. El objetivo principal del mismo tratar de descubrir cuáles pueden ser las claves del éxito de estas empresas para prosperar de una manera responsable desde la perspectiva de sus accionistas pero también de los usuarios de sus servicios aéreos y del conjunto de los recursos naturales y ambientales que constituye su base.

\section{LA RESPONSABILIDAD SOCIAL EMPRESARIAL EN SU DIMENSION SOCIAL, ECONÓMICA Y MEDIOAMBIENTAL}

La gestión y reducción del impacto medioambiental de procesos, productos y residuos, el uso sostenible de los recursos ambientales, la reducción de los riesgos ecológicos queda en manos del sector empresarial, en gran medida puesto que las actividades de producción son responsables de cuotas significativas del deterioro ambiental de nuestro planeta y de la insostenibilidad de nuestros modelos de producción y consumo. En este gran marco se integra la necesidad de generar estrategias y de acometer acciones empresariales que se dirijan al logro de aquellos objetivos; así pues, de la Responsabilidad Social Empresaria (RSE), también llamada Responsabilidad Social Corporativa (RSC).

El punto de partida es la falta de claridad acerca de si debatir acerca de la RSE/RSC se trata de una moda o consiste en una nueva forma de gestión de las organizaciones.

Analizando la evolución de la nueva idea o del nuevo concepto, se llega a comprobar la existencia de una migración de la teoría y del discurso general en los foros 
económicos y entre el público desde un concepto tradicional de empresa como aquella organización focalizada en la maximización del beneficio y en cómo repartirlo entre sus accionistas, hacia el de empresa como aquella organización de la que se espera - y a la que prácticamente se la exige - que tenga una buena relación con todos los agentes o stakeholders con los que mantiene una relación más o menos directa, tales como los trabajadores, los clientes, el medio ambiente, etc.

World Business Council for Sustainable Development define la RSE como "el compromiso que asume una empresa para contribuir al desarrollo económico sostenible por medio de la colaboración con sus empleados, sus familiares, la comunidad local y la sociedad en pleno, con el objeto de mejorar la calidad de vida" (WBCSD, 2011).

También merece la pena tener en cuenta la definición del término RSE que aporta el Centro Andaluz para la Excelencia en la Gestión (2008), dentro del Congreso "La Gestión Ambiental en el Marco de la Responsabilidad Social Empresarial" celebrado en Sevilla. Se entiende como RSE "la integración voluntaria, por parte de las empresas, de las preocupaciones sociales y medioambientales en sus operaciones comerciales y sus relaciones con sus interlocutores: accionistas, proveedores, clientes, trabajadores, administraciones y comunidades locales".

La dimensión medioambiental o, en otras palabras, la consecución de lo que se ha dado en llamar la empresa sostenible se convierte, como podemos observar, en un elemento definitivamente necesario ambas visiones acerca de la RSE.

No existe unanimidad de opiniones a la hora de plantear una definición de RSE; por el contrario, podría decirse que hay tantas definiciones como autores han tratado la materia. En lo que están casi todos de acuerdo es en que la RSE integra varios aspectos que se denominan con diferentes términos tales como enfoques, gestiones, dimensiones, etc. En concreto, existe un acuerdo generalizado de que la RSE es un conjunto complejo formado por las tres dimensiones tradicionales de la sostenibilidad: social, económica y medioambiental.

Así, la dimensión económica de la RSE se basa fundamentalmente en el Buen Gobierno Corporativo, o manera en que las empresas son gestionadas y supone velar por los intereses de los accionistas e inversores como consecuencia de la buena gestión. A este respecto, la Global Reporting Iniciative (GRI), organización sin ánimo de lucro con sede en Ámsterdam (Holanda), fue una de las primeras en el desarrollo de informes de sostenibilidad. Según un trabajo realizado por esta organización en el año 2006 sobre el sector de transporte, el impacto de esta dimensión económica se concreta en aspectos como la información económico-financiera transmitida a los accionistas.

La dimensión social de la RSE se compone de una serie de cuestiones relacionadas con la denominada cuestión social que se encarga de gestionar la actuación con los individuos. Se trata de establecer, acatar e impulsar aspectos relacionados con los derechos humanos, los derechos laborales, la protección del consumidor, la salud y seguridad en el trabajo, los proyectos de acción social, etc. En el informe de la GRI (2006) respecto del sector del transporte, se analizan dentro de esta dimensión cuestiones como la creación de empleo, la importancia de los sindicatos en la empresa, las políticas de información de los empleados, la seguridad y salud de los empleados 
y los clientes, la formación de los empleados, la gestión de los derechos humanos o los mecanismos de quejas de los clientes, entre otros aspectos.

Por último, la dimensión medioambiental de la RSE se corresponde con algo más que limitarse a cumplir las regulaciones gubernamentales correspondientes en cada lugar. Se ha transformado una obligación y una necesidad en una ventaja para las empresas que han decidido desarrollar esta dimensión. Entre las acciones de mayor importancia dentro de este enfoque se encuentra la reducción y gestión del impacto medioambiental (Observatorio de Responsabilidad Social Corporativa, 2009; Navarro, 2008), también se incluye el aprovechamiento de los recursos medioambientales y los usos sostenibles de los mismos (Sarro et al., 2007), siendo otra de las acciones importante en este ámbito la minimización de riesgos ecológicos (Navarro, 2008). En el trabajo realizado sobre el sector del transporte por la GRI (2006), esta dimensión se fundamenta en el impacto que tiene la actuación de las empresas sobre los sistemas naturales vivos o inertes. También se incluye la necesidad de dar un tratamiento adecuado de los residuos producidos, analizar las fuentes de energía utilizadas, las emisiones, etc.

En este mismo sentido, y tal y como muestra la Figura 1, Vargas et al. (2009) ponen de manifiesto diversas acciones de RSE en su dimensión medioambiental, tales como la gestión y reducción del impacto medioambiental, el uso sostenible de los recursos ambientales, la preocupación por los problemas ecológicos mundiales, la reducción de los riesgos ecológicos así como la creación de valores medioambientales.

Figura 1: Acciones de RSE en su dimensión medioambiental

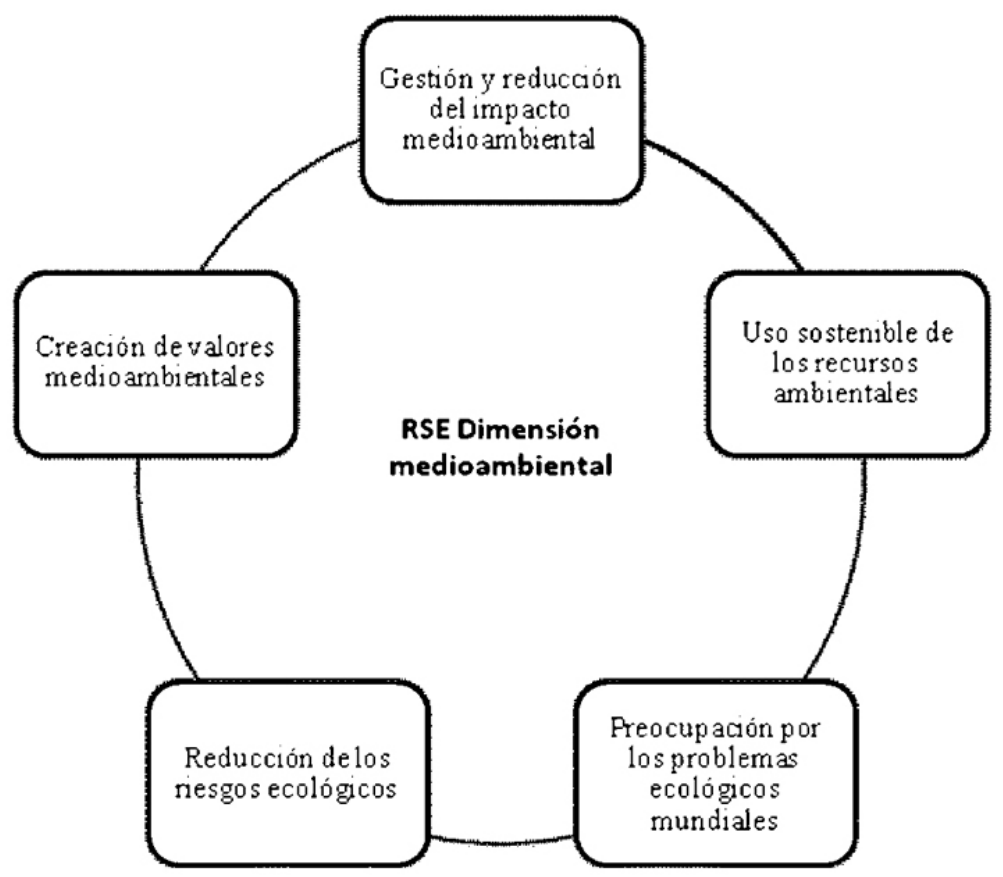

Fuente: Elaboración propia a partir de Vargas, Rodríguez y Montes (2009) 
Respecto a las acciones RSE en su dimensión medioambiental, Fernández y Merino (2005) mantienen que las actuaciones relacionadas con la reducción de la contaminación, la degradación del medioambiente o la reducción del consumo de recursos naturales no renovables son valoradas muy positivamente por la sociedad, tan solo por detrás de las actuaciones relacionadas con las condiciones de los trabajadores.

La sociedad actual, en efecto, demanda que las organizaciones reconozcan su capacidad de ocasionar serios impactos negativos sobre el medio, en sus dimensiones social, medioambiental y económica. También, que las compañías deben ser absolutamente responsables en la gestión de la actividad empresarial. Como contempla Correa (2004), "los entornos naturales sanos que tienen capacidad de renovarse, y los recursos humanos mejor educados, saludables, y motivados, son la base que asegura la continuidad de la producción y el éxito de la empresa moderna".

En este sentido, la Responsabilidad Social Empresarial (RSE) no reclama sólo la ética individual de las personas que componen las empresas, sino que ven en la organización en sí misma un ente con responsabilidad propia, económica, social y medioambiental, a la que debe exigírsele comportamientos coherentes con dicha responsabilidad.

Por consiguiente, la responsabilidad social empresarial supone que la empresa se oriente no solo hacia la maximización de los beneficios económicos, sino también al bienestar social y protección ambiental. Los indicadores de comportamiento responsable de la empresa, se analizan tanto en cuanto a las actividades externas como a las internas; se consideran en este sentido, categorías de elevada relevancia tales como la calidad de los productos y servicios que se ofertan, la creación de empleos, la capacitación ambiental, la salud y seguridad laboral, así como los programas de promoción a través de los medios, en los cuales no se deben manipular las emociones, ni distraer al público de las fuentes reales de satisfacción de sus necesidades.

Una empresa sostenible es aquella que dispone de protocolos de gestión medioambiental sobresalientes, basados en la orientación hacia la eficiencia en los procesos, la implicación de las personas en la mejora continua y en la innovación y la integración de la vertiente de sensibilización social respecto a la disminución de los impactos ambientales.

Tampoco debe olvidarse que con la incorporación de unas prácticas empresariales socialmente responsables las empresas consiguen ventajas competitivas. Así por ejemplo, la empresa líder en España en distribución, "El Corte Inglés", integra la RSE en todas las áreas de la organización y para ello cuenta con un Comité de Responsabilidad Social integrado por la alta dirección y donde están representadas todas las áreas estratégicas. Esta empresa pretende para ello llevar una relación, lo más fluida posible, con todos los grupos de interés con los que se relaciona. Esta empresa ha sido la primera empresa en disponer de plazas de aparcamiento para coches eléctricos con recarga gratuita, mostrando así un comportamiento medioambiental proactivo.

Si se observa desde una perspectiva puramente macroeconómica y global, la gestión con criterios de responsabilidad social empresarial contribuye sin duda al desarrollo sostenible y equilibrado del planeta. Pensando en las generaciones futuras y, 
tal como refleja González (2004), si lo que se persigue es generar riqueza de forma sostenible sin agotar los recursos naturales y minimizando la huella medioambiental de nuestra generación, todos tenemos que mejorar los procesos para que así sea.

Observando la importancia las tendencias a favor de la RSE y recordando la importancia del sector turístico en la economía de nuestro país y, en especial, el protagonismo de las compañías aéreas, resulta peculiar observar la existencia escasa de trabajos académicos que analicen la responsabilidad social empresarial o responsabilidad social corporativa en dicho sector, en especial desde su enfoque medioambiental. Actuaciones de esta naturaleza están estrechamente relacionadas con la actividad que desarrollan las compañías aéreas, razón por la que creemos conveniente analizar, profundizar y evaluar las acciones medioambientales llevadas a cabo por las compañías aéreas.

\section{METODOLOGIA}

Para llevar a cabo el análisis de esta investigación, se ha utilizado la técnica del Análisis de Contenido web de las compañías aéreas que operan en el territorio español. Este método de análisis requiere seguir una serie de fases secuenciales para su adecuada aplicación. En el estudio que nos ocupa, se ha procedido a realizar seis fases con las características que se describen a continuación:

- Fase I. Establecimiento de Objetivos.

Se han identificado los diferentes objetivos que se pretende alcanzar, que en el caso de esta investigación son los siguientes:

- Identificar si las páginas webs de las compañías aéreas contienen información de las actividades de responsabilidad social corporativa que realizan.

- Analizar las acciones relacionadas con el medioambiente.

- Comprobar si existen diferencias entre las distintas nacionalidades de las compañías y su implicación con el medioambiente.

- Fase II. Selección de la muestra

Seleccionar la muestra de las compañías que se van a estudiar de forma que resulte representativo del total de compañías que operan en España. Para realizar esta selección se ha buscado información sobre las compañías aéreas que mayor número de viajes realizan tanto de entrada en como de salidas de España. Durante el año 2007, de los viajeros que llegaron a España por vía aérea, más del65 por ciento lo hizo en alguna de las veinte compañías principales (IET, 2010).

La muestra que se ha utilizado en esta investigación está compuesta por las 50 compañías que más viajeros transportan, que representan más del 95 por ciento del tráfico aéreo de España (AENA, 2009). Es decir, de los 202 millones de viajeros que utilizaron este medio de transporte para sus desplazamientos, más del 95 por ciento utilizó alguna de las compañías analizadas.

- Fase III. Definición de las variables.

Se han definido las variables que se han utilizado para poder alcanzar los objetivos fijados. Las variables vienen definidas en el apartado de análisis de resultados. 
- Fase IV. Definición de la codificación.

En esta fase se desarrolló un manual de codificación que permitiera a todos los codificadores evaluar las variables según las categorías establecidas y junto a las instrucciones necesarias indicadas en dicho manual, poder codificar toda la información de manera homogénea.

- Fase V. Codificación de las variables.

En esta fase se procedió a la codificación de las variables según lo establecido en el apartado anterior y para lo cual, los codificadores se instruyeron previamente. En este trabajo han participado las autoras de la investigación y el nivel de coincidencia entre ellas ha sido elevado.

- Fase VI. Análisis cualitativo y descriptivo.

En esta última fase se realizó un análisis cualitativo y un tratamiento estadístico de los datos obtenidos, realizándose con ellos un análisis descriptivo que ha permitido llegar a una serie de conclusiones.

\section{RESULTADOS Y ANALISIS}

La primera observación que debemos plantear, después de analizar cuidadosamente las distintas páginas web de las principales compañías aéreas españolas, es la dificultad que entraña encontrar la información relacionada con las políticas de responsabilidad social de las empresas en cualquiera de sus ámbitos, en la mayor parte de los casos y, en concreto, en el ámbito de actividad que nos ocupa. En este sentido, solamente el $17,8 \%$ de las webs analizadas contiene una memoria de RSE. Este dato resulta interesante a la vista de la importancia de estas actuaciones en la estrategia empresarial en la actualidad y, en concreto, en el sector del transporte aéreo; sin embargo, conviene señalar que la no existencia de documentos así denominados no supone que no se hayan realizado acciones de RSE aunque su ausencia genere en el equipo de investigación alguna preocupación sobre el grado de compromiso de estas empresas con la RSE en sí misma.

Sí tenemos en cuenta la naturaleza de las compañías aéreas, se observa que existen diferencias más que significativas entre las compañías tradicionales y las de bajo coste, desde la perspectiva de la identificación de aquellas que disponen de memoria RSE. Como hemos podido comprobar, disponen de memoria RSE todas compañías tradicionales analizadas y, por el contrario, ninguna de las compañía de bajo coste (ver cuadro 1). Esto nos lleva a plantear la cuestión de si la reducción de los costes estará directamente relacionada con menores grados de preocupación en materia social o si, por el contrario el compromiso y el trabajo en esta dirección existe aunque no se haya incorporado a cada página web de las aerolíneas en particular. Como hemos comentado anteriormente, que no exista memoria RSE no significa que no se desarrollen acciones a favor del medioambiente o que se trate de ser compañías no comprometidas ni preocupadas por la sostenibilidad. 
Cuadro 1. Existencia de memoria de RSE

\begin{tabular}{|c|c|c|c|}
\hline Existencia memoria & Compañías Tradicionales & Compañías Bajo Coste & Total \\
\hline $\mathrm{Si}$ & 23,5 & 0 & 17,8 \\
\hline $\mathrm{No}$ & 76,5 & 100 & 82,2 \\
\hline
\end{tabular}

Fuente: Elaboración propia a partir de los datos obtenidos de las webs de las aerolíneas

La prueba de asociación "chi cuadrado" de Pearson para la existencia de memoria RSE, según el tipo de compañía muestra los valores que se recogen a continuación:

\begin{tabular}{|c|c|c|}
\hline Chi cuadrado & Grados de libertad & Significación \\
\hline 3,254 & 1 & 0,068 \\
\hline
\end{tabular}

\subsection{ACCIONES PROAMBIENTALES REALIZADAS POR LAS COMPAÑÍAS AÉREAS}

Los resultados obtenidos muestran como las compañías aéreas centran casi toda su atención en unas acciones medioambientales muy concretas relacionadas fundamentalmente con la intención de disminuir las emisiones de dióxido de Carbono (CO2).

Las emisiones de dióxido de carbono afectan directamente al cambio climático con efectos relevantes e irreversibles para el planeta, hecho por el cual se está trabajando intensamente para tratar de reducir, controlar y compensar esas emisiones. Según el Panel Intergubernamental contra el cambio climático el sector que estamos estudiando emite el $2 \%$ del total de emisiones y estos, al lado de las emisiones de otros gases que surgen de la combustión de sus motores y de la contaminación acústica que generan, conducen a considerar que este sector es muy significativo en términos de participación en el origen de la contaminación y de los problemas ambientales globales. La Unión Europea se destaca en aplicar políticas de mitigación de gases de efecto invernadero procedentes del sector aéreo, como pone de manifiesto la Directiva 2008/101/CE del Parlamento Europeo y del Consejo que somete al Régimen Europeo de Comercio de Emisiones las de $\mathrm{CO}_{2}$ de las aeronaves que atraviesan el espacio aéreo europeo aterrizando o despegando de aeropuertos comunitarios.

En el sector empresarial de la aviación existen diferentes alternativas para tratar de disminuir la contaminación que se produce por las emisiones de $\mathrm{CO}_{2}$. Las acciones más habituales encontradas tras el análisis de las páginas webs, podemos clasificarlas en dos grandes clases: medidas operacionales y actuaciones para la renovación de la flota de aviones.

Entre las medidas operacionales cobran mayor relevancia las relacionadas con la utilización de motores más eficientes y el logro de una mejor aerodinámica. La reducción del peso de los aviones está directamente relacionada con un menor consumo de combustible y, en consecuencia, genera reducciones en las emisiones de dióxido de carbono.

Existe una tercera medida operacional en la que las compañías están invirtiendo esfuerzos considerables en la actualidad: la utilización de biocombustibles como carburante de los motores tradicionalmente alimentados por queroseno, de origen fósil. Los biocombustibles se producen de manera orgánica a partir de diversas especies de uso agrícola y se les atribuye al quemarse un GWP igual a cero (igual al saldo de 
absorciones menos emisiones de $\mathrm{CO}_{2}$ de los materiales agrícolas o forestales de que proceden, ya que al crecer absorbieron dióxido de carbono). Sin embargo, existen detractores a este tipo de combustible por los posibles efectos nocivos que pueden tener para el suelo fértil o en términos de utilización de la superficie agraria útil del planeta, en competencia con la producción de alimentos para la población mundial. Por ello, las investigaciones más avanzadas en el sector de la aviación se dirigen a la utilización de algas como input en la producción de biocombustibles.

De las diferentes acciones a favor del medio ambiente realizadas por las compañías aéreas analizadas, las relacionadas con la reducción de $\mathrm{CO}_{2}$ a través de medidas operacionales son precisamente las que aparecen en un mayor número de casos. El $57,8 \%$ de las acciones realizadas por dichas compañías son de esta naturaleza.

La segunda clase de medidas contempladas, dentro de las diferentes alternativas para tratar de disminuir las emisiones de $\mathrm{CO}_{2}$, es la renovación de la flota. Su importancia relativa no es excesiva y en las compañías analizadas, adquiere una participación del 17,8\%. Con esta medida, en los últimos años se ha logrado reducir las emisiones, por las mejoras tecnológicas incluidas en los motores y el ahorro en el consumo de combustible debida a la utilización de reactores. Existen diferencias significativas entre las compañías tradicionales y las de bajo coste, en lo relativo a este particular tipo de acciones; en efecto, ninguna de las compañías de bajo coste recoge entre sus iniciativas la de renovar su flota de aviones para tratar de reducir las emisiones de $\mathrm{CO}_{2}$.

Figura 2. Renovación de la flota para reducir emisiones de $\mathrm{CO}_{2}$

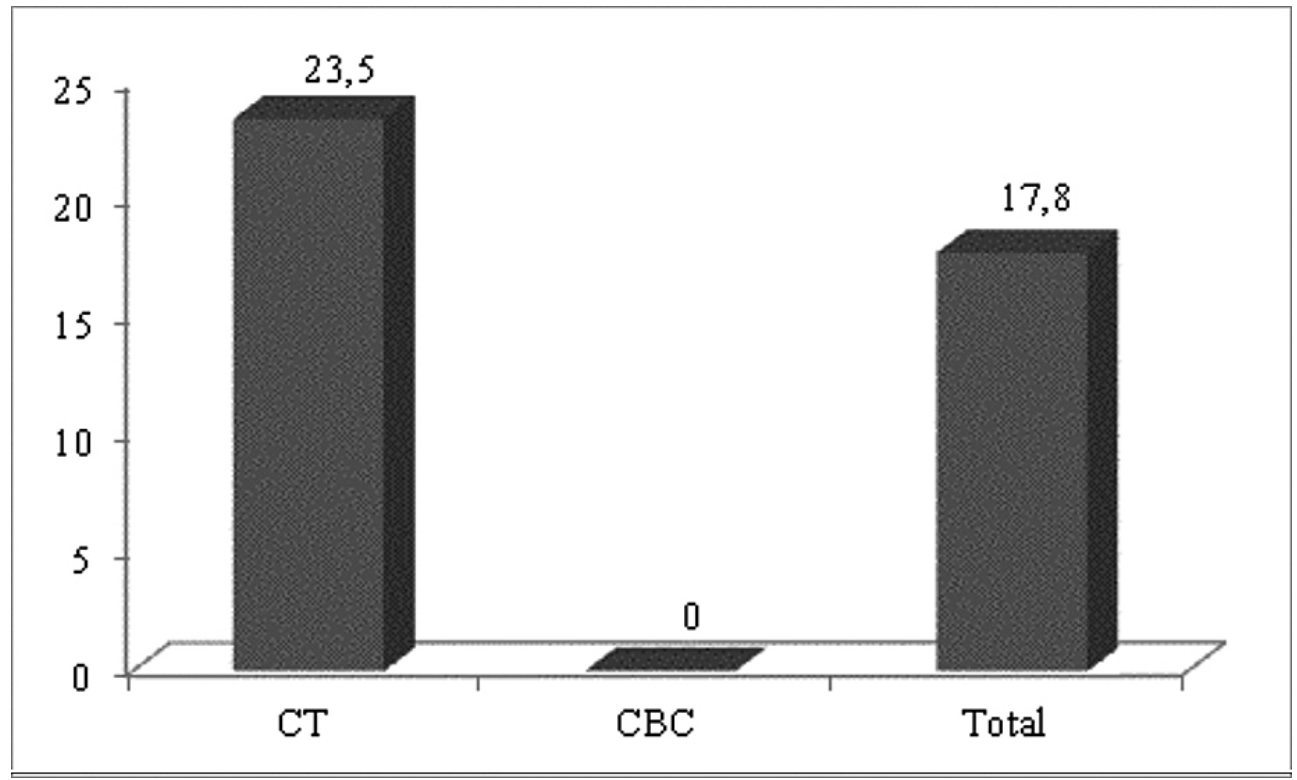

Fuente: Elaboración propia a partir de los datos obtenidos de las webs de las aerolíneas

A medio camino en la importancia relativa de estas dos medidas relacionadas con la reducción de emisiones de dióxido de carbono, se encuentra una actuación que 
concierne al ruido generado por los aviones. La contaminación acústica de este origen y de la que es responsable el sector de la aviación, se localiza en las zonas próximas a los aeropuertos; se deriva de las acciones de ascenso, despegue, aproximación y aterrizaje de los aviones a las pistas. Para tratar de minimizar este efecto las compañías aéreas están introduciendo mejoras aerodinámicas e incorporando avances que permitan reducir el ruido que produce cada avión. Estas acciones representan el $26,7 \%$ de las realizadas por las compañías analizadas.

La siguiente medida a tener en cuenta, a favor del medio ambiente, corresponde a la reducción en el consumo de agua. En las compañías aéreas, el consumo de agua viene exigido por numerosas actividades de mantenimiento de las aeronaves. Para intentar reducir este consumo se están utilizando últimamente aguas residuales que se tratan a través de depuradoras, disminuyendo pues la presión sobre recursos hídricos para usos consuntivos o de riego. De nuevo en este apartado existen diferencias muy significativas entre las compañías tradicionales y las de bajo coste. En las páginas webs de éstas últimas no se ha identificado ninguna compañía que recogiera entre sus acciones el ahorro de agua como política medioambiental a desarrollar.

Figura 3. Acciones medioambientales en el sector aéreo

$\because \mathrm{CBC} \equiv \mathrm{ET}$

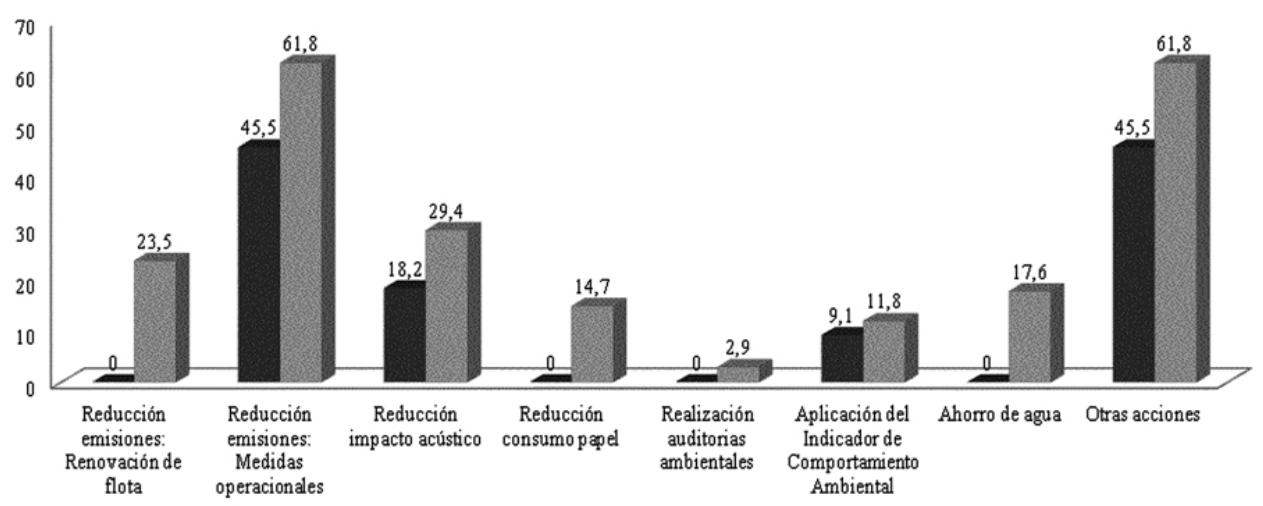

Fuente: Elaboración propia a partir de los datos obtenidos de las webs de las aerolíneas

Figura 4. Acciones medioambientales en el sector aéreo según tipo de compañía aérea

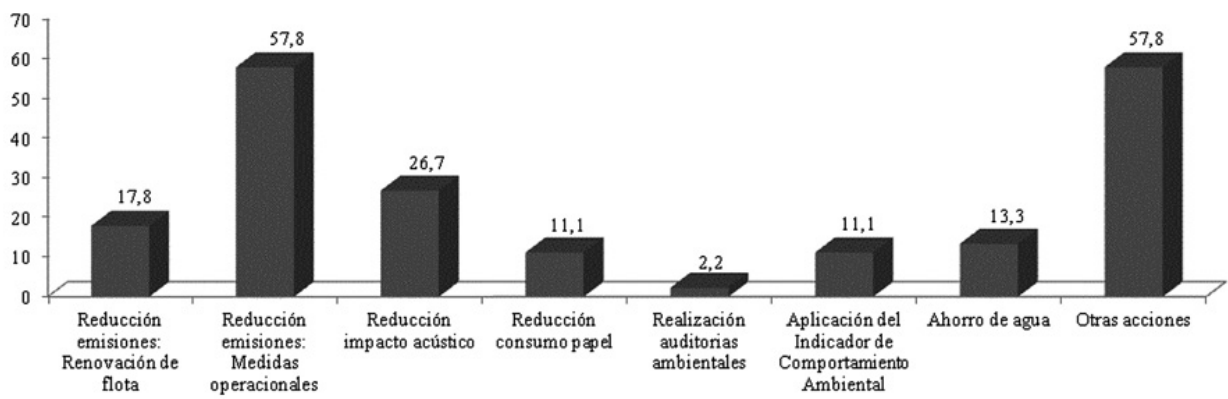

Fuente: Elaboración propia a partir de los datos obtenidos de las webs de las aerolíneas 
También, en menor medida, las compañías aéreas que desarrollan algunas otras actuaciones en este mismo sentido. Ellas se recogen en la Figura 4 en el apartado de Otras, y pueden identificarse una cantidad limitada pero variopinta de propuestas proambientales realizadas por las aerolíneas, tales como: programas de reciclaje de papel, plástico y otras materias, campañas dirigidas a sensibilizar a los empleados de la necesidad de desarrollar conductas que permitan hacer un buen uso de los recursos y una gestión adecuada de los residuos, etc.

Por último, es preciso aludir al hecho de que la limpieza de los aviones no está relacionada únicamente con el consumo de agua, sino que también se utilizan productos de limpieza que producen unos residuos nocivos. Para minimizar su impacto negativo, se propone el uso de productos biodegradables, si bien es cierto que esta medida aparece en un número muy reducido de casos.

\section{CONCLUSIONES}

La Responsabilidad Social Empresarial (RSE), también llamada Responsabilidad Social Corporativa (RSC), es un concepto relativamente novedoso y uno de los más complejos retos que la gestión empresarial enfrenta en nuestros días. El sector aéreo que proporciona servicios de transporte a un gran número de turistas y genera no despreciables volúmenes de actividad y empleo en España no es ajeno a este compromiso. El desarrollo sostenible desde su triple perspectiva: económica, social y medioambiental así lo exigen.

Para poder alcanzar el cambio organizacional necesario mediante un comportamiento empresarial verdaderamente responsable y llevar a cabo estas políticas, debe existir por parte de la sociedad una fuerte motivación para el cambio, una percepción de la necesidad del cambio, un planteamiento de objetivos claros y precisos, la existencia de liderazgo y agentes internos y externos motores del cambio, planes, programas de acción y mecanismos de control con respecto al proceso de cambio y, finalmente el análisis y la evaluación de sus resultados.

Desarrollar un análisis de la dimensión medioambiental de las compañías aéreas que operan en España, requiere una cuidadosa observación y estudio del contenido de estas compañías en sus páginas web. Pues bien, se han encontrado grandes dificultades a la hora de localizar información relacionada con las políticas de RSE en el ámbito que nos ocupa, lo que es una realidad constante en nuestra labor de análisis de la realidad. Tal y como hemos contemplado a lo largo de esta exploración, es incuestionable la necesidad de que las compañías aéreas de aumenten y mejoren la información que ofrecen, con respecto a este ámbito de actividad.

A pesar de las citadas barreras se han obtenido resultados que muestran como las compañías aéreas centran casi toda su atención en unas acciones medioambientales muy concretas. La pretensión de disminuir las emisiones de $\mathrm{CO}_{2}$, la de reducir el consumo de agua, los programas de reciclaje de papel, plástico y otras materias, las campañas dirigidas a sensibilizar a los empleados de la necesidad de desarrollar conductas que permitan hacer un buen uso de los recursos y una gestión adecuada de los residuos, así como las acciones dirigidos a el uso de productos biodegradables, son 
políticas frecuentes de las compañías aéreas, en el periodo analizado. Estas pueden considerarse acciones y políticas de RSE desde el punto de vista medioambiental pero, como hemos podido observar, solo se llevan a cabo en las empresas tradicionales y, en pocas ocasiones, en compañías aéreas de bajo coste, lo que es una realidad preocupante como hemos reflejado anteriormente.

A pesar de todos los esfuerzos, programas, estrategias y experiencias concretas de responsabilidad social empresarial que se han puesto en marcha, éstas no resultan aún suficientes para avanzar significativamente hacia la solución de la actual crisis socio-ambiental a la que se enfrenta nuestro sistema socioeconómico y natural. Para alcanzar el objetivo de una verdadera sostenibilidad del modelo de desarro1lo, resulta imprescindible la actuación desde una perspectiva general, orientada precisamente hacia el cambio estructural y funcional del actual sistema social, económico y político predominante.

El éxito de la RSE/RSC, tanto en el ámbito concreto de las compañías aéreas que operan en España - tomado como objeto de análisis en la presente investigacióncomo en general, en todos los sectores de actividad, sería conseguir que las empresas encontraran el camino para prosperar de una manera responsable, trabajando con otros actores sociales y económicos para lograr modificaciones en el sistema económico en la dirección adecuada, es decir en pro del desarrollo sostenible.

Dentro del ámbito concreto al que este estudio se ha dedicado se propone, a la vista de los resultados obtenidos, la profundización de los mismos y el inicio de otras líneas de investigación colaterales sobre RSE/RSC mediante distintas técnicas, como entrevistas en profundidad, encuestas o reuniones de grupo a accionistas, usuarios, proveedores, etc.

\section{BIBLIOGRAFÍA}

CENTRO ANDALUZ PARA LA EXCELENCIA EN LA GESTIÓN (2008). La Gestión Ambiental en el marco de la Responsabilidad Social Empresarial. Instituto Andaluz de Tecnología.

CORREA, M.E. (2004). "Responsabilidad social empresarial: una nueva forma de hacer negocios". Revista Futuros, No. 6. Vol. 2.

EXCELTUR (2009): "Perspectivas Turísticas. Valoración empresarial del año 2008 y perspectivas para 2009”. Exceltur-Alianza para la excelencia turística, $\mathrm{N}^{\mathrm{o}} 27$, enero, pp. 4.

FERNÁNDEZ, D.; MERINO, A. (2005). “ ¿Existe disponibilidad a pagar por responsabilidad social corporativa? Percepción de los consumidores". Universia Business Review, $\mathrm{n}^{\circ}$ 7, pp 38-53.

FIGUEROLA, M. (2011). "La Aportación del Turismo al PIB Español". Nexotur, www.nexotur.com. URL consultada el 28 de Septiembre 2011.

GLOBAL REPORTED INICIATIVE (2006a), Guía para la elaboración de Memorias de

Sostenibilidad, http://www.globalreporting.org/NR/rdonlyres/415F297B-5289-41608B6C7CC034D5BE52/0/G3_GuidelinesESP.pdf URL consultada el 28 de Septiembre 2011. 
GONZÁLEZ, M. (2004). El porqué de la responsabilidad social corporativa. BOLETÍN ECONÓMICO DE ICE Nº 2813.

INSTITUTO DE ESTUDIOS TURÍSTICO-IET (2007), Turismo, tráfico aéreo y Compañias Aéreas de Bajo Coste 2006, Secretaría de Estado de Turismo y Comercio, Madrid.

INSTITUTO DE ESTUDIOS TURÍSTICO-IET (2010), Encuesta de Estudios Turísticos en Fronteras -Frontur. Ministerio de Industria, Turismo y Comercio, Madrid.

INSTITUTO DE ESTUDIOS TURÍSTICO-IET (2011A), Encuesta de Estudios Turísticos en Fronteras -Frontur. Ministerio de Industria, Turismo y Comercio, Madrid.

INSTITUTO DE ESTUDIOS TURÍSTICO-IET (2011b), Encuesta de Gasto Turístico en Fronteras -Egatur, Ministerio de Industria, Turismo y Comercio, Madrid.

INE (2011). http://www.ine.es/ URL consultada el 5 de Octubre de 2011.

NAVARRO, F. (2008), Responsabilidad Social Corporativa: Teoría y práctica. ESIC. Madrid.

OBSERVATORIO DE RESPONSABILIDAD SOCIAL CORPORATIVA (2009), $\mathrm{http}: / /$ www.observatoriorsc.org/index.php?option $=$ com content\&task=view\&id $=40 \&$ Itemid $=6$, URL consultada el 16 de marzo de 2009 .

PÉREZ, J.M. (2011). "Compañías que más pasajeros transportaron en cada aeropuerto en 2010". Avion Revue, $\mathrm{n}^{\mathrm{o}}$. 351.

REVISTA FUTUROS. (2011). Responsabilidad Social Empresarial. American Friends Service Committee. http://www.revistafuturos.info/futuros17/resp_soc_emp.htm URL consultada el 16 de septiembre de 2011.

SARRO, M.M.; CUESTA, P.; PENELAS, A. (2007). "La Responsabilidad Social Corporativa (RSC) una orientación emergente en la gestión de las entidades bancarias españolas". Conocimiento, Innovación y Emprendedores. Camino al futuro. Universidad de la Rioja.

VARGAS, A.M.; RODRÍGUEZ, B.; MONTES, Mª P. (2009). “Análisis de contenido de las webs de las compañías aéreas: acciones de responsabilidad social corporativa". Administrando en entornos inciertos. ESIC Editorial.

WORLD BUSINESS COUNCIL FOR SUSTAINABLE DEVELOPMENT (WBCSD), (2011). http://www.wbcsd.org. URL consultada el 16 de septiembre de 2011. 